\section{Martin Fey}

Klinikdirektor und Chefarzt der Universitätsklinik für Medizinische Onkologie, Inselspital und Universität Bern

Der Artikel gibt die persönliche Meinung des Autors wieder. Er entspricht nicht einer offiziellen Stellungnahme des Inselspitals.

Eine Replik von Jürg Nadig auf diesen und den folgenden Artikel erscheint in der nächsten Ausgabe.

Korrespondenz:

Prof. Dr. med. Martin Fey

Universitätsklinik für

Medizinische Onkologie

Inselspital und Universität Bern

CH-3010 Bern

martin.fey[at]insel.ch
In der SÄZ 4/2013 [1] erschien unter dem dramatischen Titel «HSM Onkologie: Speerspitze einer Zentralisierung der ambulanten Versorgung» ein Pamphlet von Jürg Nadig (dem Präsidenten der Schweizerischen Gesellschaft für Medizinische Onkologie SGMO) und sieben weiteren Autoren. Das Fähnlein dieser acht Aufrechten geisselt den Bericht einer Expertengruppe zur HSM Onkologie. Das HSM-Dokument legt, basierend auf der Interkantonalen Vereinbarung zur Hochspezialisierten Medizin (IVHSM), Vorschläge zur Zentralisierung der Versorgungsstruktur der Onkologie in der Schweiz vor; der Versand in die Vernehmlassung erfolgte Mitte Dezember 2012.

Die im HSM-Bericht angedachte Bildung von sieben Comprehensive Cancer Centres (CCC) wird von Nadig et al. als «kartellistische Aufteilung» der Versorgung von Krebspatienten in der Schweiz gebrandmarkt. Die übrigen onkologischen Leistungserbringer würden ohne Grund beschnitten, die freie Arztwahl der Krebspatienten werde grundlos eingeschränkt. Diese und weitere Vorwürfe fallen - mit anderen Worten, es droht scheinbar onkologische Apocalypse Now! Aufregung und rühriges Aufbegehren der Kreise um Jürg Nadig beschränken sich nicht auf diesen Diskussionsbeitrag in der SÄZ. Im Zürcher Tagesanzeiger kritisiert Thomas von Briel, frei praktizierender Onkologe in der Region Zürich, mit der HirslandenGruppe affiliert, das für den HSM-Bericht verantwortliche Fachorgan, und er wirft prominenten Schweizer Onkologen, Prof. Thomas Cerny aus St. Gallen und Prof. Franco Cavalli aus dem Tessin, vor, ihre Mitarbeit am Bericht zur Wahrung ihrer Eigeninteressen missbraucht zu haben. Ich erhalte in den letzten Tagen aus diversen Quellen ein regelrechtes E-Mail-Gewitter zum Thema HSM Onkologie auf meinem Computer-Bildschirm. Nadig selber ruft dazu auf, den «Irrweg der HSM Onkologie» mit einem Twitter anzuprangern, mithin wird anti-zentralistisch gefacebooked und die Kommunikationstechnik pubertierender Jugendlicher gekonnt übernommen. Swiss Oncology goes bonkers!

Was steht denn Schlimmes im HSM-Bericht? Er schreibt in der Präambel, die aktuelle Versorgungslage für Krebspatienten in der Schweiz sei nicht immer optimal; in einer dezentralen, föderalistisch geprägten Struktur sei die Kooperation der Leistungserbringer oftmals personenabhängig und $\mathrm{zu}$ wenig koordiniert. Das stimmt doch zweifelsohne, oder etwa nicht? Vor diesem Hintergrund hat das HSMFachorgan nun Empfehlungen zur Konzentration gewisser seltener Tumoren auf wenige Zentren erarbeitet, um dadurch die Koordination und Kohärenz der Krebsbehandlung in der Schweiz zu verbessern. Was soll denn an dieser Idee so verwerflich sein? Es ist schwer verständlich, weshalb die strukturiert-verbindliche Vernetzung in der Betreuung von Patienten mit malignen Hirntumoren, mit Sarkomen (in all ihren Unterformen selten bis sehr selten), oder malignen neuro-endokrinen Tumoren mit sieben grossen Zentren in der Schweiz so viel Widerstand und aufmüpfige Protestaktionen nach sich zieht.

Die Idee, mit CCCs klinische Centres of Excellence in der Schweiz zu kreieren, ist weder ein Novum noch eine unfundierte Schnapsidee der HSMGruppe. Das Ausland lebt uns füglich vor, wo und wie hervorragende Krebsmedizin praktiziert wird, für seltene und für häufige Tumoren. Nicht im onkologischen Einzelstübli in Bülach, sondern am Dana Farber Cancer Center in Boston, am Institut Gustave Roussy in Paris und anderswo. Wenn die Deutsche Krebsgesellschaft (ehemals Mildred-Scheel-Stiftung) nicht gute Gründe hätte, in Deutschland Millionenbeträge in die Weiterentwicklung grosser deutscher Krebszentren zu pumpen, würde sie ihre Mittel wohl anders einsetzen. Dass die Betreuung von Krebspatienten in Spitälern mit höherem Case load bessere Resultate erzielt als die Behandlung in kleinen Institutionen mit geringeren Fallzahlen, ist für diverse Tumortypen belegt (s. Literatur am Ende). Nadig zitiert jedoch eine Studie zur Behandlung des HodgkinLymphoms, der er entnimmt, die Behandlung dieser Krankheit sei an kleineren Zentren oder bei niedergelassenen Onkologen Deutschlands gleich gut wie in CCCs [Klimm et al., 2]. Sein Zitat und seine Erläuterung sind aber unvollständig: wesentlich an der Klimm-Analyse war, dass alle Patienten in den Deutschen Hodgkin-Studien behandelt wurden - damit oblag dem Studien-Zentrum in Köln (einem CCC, oh Schreck!) die Sicherung der Qualitätskontrolle auch in der Peripherie. Der argumentative KlimmZug von Jürg Nadig leitet demnach bei näherer Betrachtung eher Wasser auf meine Mühle. Qualitätskontrolle durch ein grösseres Zentrum in seinem Wirkungsbereich ist just ein Ziel des Projekts HSM Onkologie - Widerstände dagegen sind meines Erachtens schwer einsehbar.

Die Zentralisierung der Krebspatientenbetreuung bringt nämlich nicht nur Vorteile dank besserer Vernetzung zahlreicher Spezialisten in einer Institution (was in dieser Form beispielsweise am Spital Männedorf, aus dem einer der Ko-Autoren stammt, eben gar nicht denkbar wäre), sondern fördert auch klinische Forschung. Zu viele Probleme in der Onkologie sind ungelöst, zu viele sog. «Standards» oder Routinetherapien unbefriedigend, als dass die Schweizer Krebspatienten auf den Zugang zu klini- 
schen Protokollen verzichten sollten. Die gesetzlichen Grundlagen zur Durchführung klinischer Studien in der Schweiz verlangen, dass die Forscher (investigators) einen grossen Anteil an direkter Verantwortung für Studienpatienten übernehmen. Die logistischen Anforderungen an die Durchführung klinischer Studien nach Massgabe von Good clinical practice guidelines können von vielen Einzelpraxen und kleineren Spitälern nicht erfüllt werden; mit Einführung des neuen Humanforschungsgesetzes (HFG) wird dies nicht entschärft, sondern noch

\section{Zu viele Probleme in der Onkologie sind ungelöst, als dass Patienten auf den Zugang zu klinischen Protokollen verzichten sollten.}

straffer. Gerade wegen der problematischen Verzettelung der medizinischen Betreuung in der Schweiz hat unser Land in der klinischen Forschung nicht den Platz, den es, analog zur experimentellen Krebsforschung, haben könnte. Wenn die gegenwärtigen Strukturen in der Schweiz für klinische Forschung, gerade bei seltenen Tumoren, ungenügend sind, so ist Zentralisierung mit regionaler Vernetzung das einzig Richtige, auch wenn sie Widerstände zeugt. Bezeichnenderweise wird im Artikel von Nadig und seinen Mitstreitern die klinische Forschung mit keinem Wort erwähnt. Nadig selber hat laut PubMed knapp ein halbes Dutzend wissenschaftliche Publikationen aufzuweisen, die letzte im vergangenen Millenium, Thomas Cerny dagegen fast 200, und Franco Cavalli über 400. No further comments required.

Überhaupt: Wer ist denn auf der Seite Nadigs? Er zitiert gerne seine «repräsentative» Umfrage unter den Mitgliedern der SGMO, die sich mit 98,5\% der Stimmen vernichtend gegen das HSM-OnkologieKonzept geäussert habe. Ganze 54 Voten aus der gesamten Schweiz (in unserem Land arbeiten etwa 30000 Ärzte!) gingen bei ihm ein, davon lediglich eines aus einem Universitätsspital (wohl mein eigenes, seine einzige Gegenstimme). Mehr als die Hälfte der Voten stammt aus dem Kreise privat praktizierender Onkologen, die wohl im Schulterschluss mit Nadig um ihre Pfründen bangen, wenn sie kein Thymuskarzinom oder kein Osteosarkom mehr behandeln dürften. Ist eine derartige Umfrage wirklich «repräsentativ» für die Stimmen der Krebskliniker in der Schweiz, zu denen ja auch die Chirurgen, die RadioOnkologen, die Gynäkologen und viele andere zählen, die Nadig über die SGMO-Adresskartei wohl gar nicht erreicht hat. Representative ? ... rather not! ... Eine Meinungs-manipulative Darstellung - das ja.

Schliesslich wird im Speerspitzen-Artikel darauf hingewiesen, wie gut die Qualität der dezentralisierten Krebsbetreuung in der Schweiz ohnehin schon sei, und dass ein von Nadig gegründetes Swiss Cancer
Network garantiere, dass Schweizer Krebspatienten nach internationalen Standards behandelt würden; das Schweizerische Krebs-Netzwerk koordiniere «Aktivitäten auf verschiedenen Versorgungsebenen von der Vorsorge bis zur Behandlung». Zum Swiss Cancer Network habe ich mich bereits im Schweizerischen Krebs-Bulletin geäussert [3]. Antworten auf meine Kritikpunkte habe ich nie erhalten. Es besteht im Wesentlichen aus einem losen Verbund von ca. 80 Schweizer Onkologen (mehrheitlich medizinische Onkologen aus der Praxis; keine Chirurgen, keine Gynäkologen, praktisch keine Radio-Onkologen, keine Pflege), die angeblich Daten in dieses Register eingeben (werden), was für Qualität bürgen soll. Der denkbar oberflächlich gehaltene Text der Website www.swisscancernetwork.ch gibt kaum Auskunft, wie eingegebene Daten kontrolliert würden (beispielsweise durch Überprüfung der Original-Krankengeschichte als source document), und wie genau die klinische Qualität der gemeldeten Behandlungsfälle gemessen würde. Trotzdem erhält, wer mitmacht, ein Qualitätszertifikat - ein Billig-«Label», wenn ich daran denke, welch enormen Aufwand wir am Inselspital bieten mussten, um Qualitätszertifikate für unser Stammzell-Hochdosis-Programm oder für unser Brust- und Gynäkologie-Tumorzentrum zu erhalten. Realiter ist das Swiss Cancer Network derzeit nicht operativ tätig, und es ist nicht statthaft, es aktuell als gesetztes Qualitätssicherungsinstrument zu zitieren.

Der Eigennutz, der den Herren Cerny und Cavalli in der Sache HSM Onkologie vorgehalten wird, ist wohl eher im Nadig-Lager zu orten; denn Sachargumente gegen die HSMKonzepte bei seltenen onkologischen Erkrankungen gibt es wohl kaum. Es bleibt zu hoffen, dass die politischen Stellen, die Behörden, die Gesundheitsdirektoren-Konferenz und andere wichtige Organe auch zwischen den Zeilen der NadigTruppe lesen können. Dass es schliesslich nicht Exklusivaufgabe der Präsidenten von FMH-Fachgesellschaften ist, einseitig, teils manipulativ, teils verdeckt, die Interessen der frei praktizierenden Kollegen zu vertreten, sei zum Abschluss angemerkt.

\section{Literatur}

1 Nadig J et al. HSM Onkologie: Speerspitze einer Zentralisierung der ambulanten Versorgung? Schweiz Ärztezeitung. 2013;94(4):94-6.

2 Klimm et al. Einfluss von onkologischen Behandlungszentren auf den Therapieerfolg beim Hodgkin-Lymphom Dtsch Ärztebl Int. 2012;109(51-52): 893-9.

3 Fey M. A letter to the President of the newly founded Swiss Cancer Network. Schweiz. Krebsbulletin. 2012; 32:194-5.

\section{Literatur zur Frage des Zentrumnutzens}

- Breast 2012; 21: 261 (Mammakarzinom); Ann Intern Med 2003; 139: 649, und Dan Med J 2012; 59: B4428 (Kolonkarzinom); Int J Colorectal Dis 2012; Epub (Rektumkarzinom); Gynecol Oncol 2009; 115: 199, und Int J Gynecol Cancer 2009; 19: 94 (Ovarialkarzinom); Acta Oncol 2012; 51: 706 (Weichteilsarkome); Ann Oncol 2013; Epub (Pädiatrische Onkologie); Blood 2005; 105: 2979 (Stammzelltransplantation); J Natl Cancer Inst 1987; 78: 471 (Hodgkin-Lymphom). 